\title{
Dolor en amigdalectomía: Técnica fría versus electrobisturí monopolar. Estudio prospectivo randomizado
}

\author{
Postoperative tonsillectomy pain: Cold versus monopolar electrocautery \\ technique. Prospective randomized trial
}

Santiago Cornejo $\mathbf{S}^{1}$, Constanza Beltrán $\mathbf{M}^{1}$, Hayo Breinbauer $K^{1}$, Ximena Fonseca $\mathbf{A}^{1}$, Ramón Serra $\mathrm{T}^{2}$.

\section{RESUMEN}

Introducción: Existen diferentes técnicas para realizar la amigdalectomía. Dentro de las más frecuentes está la técnica fría (bisturí frío, tijera y asa), y la técnica con electrobistrurí monopolar. No hay consenso que alguna de estas técnicas sea claramente superior a la otra respecto al dolor posoperatorio.

Objetivo: Evaluar si la técnica quirúrgica (técnica fría-electrobisturí monopolar) repercute sobre la percepción de dolor posoperatorio.

Material y método: Ensayo clínico prospectivo, ciego y randomizado. Se incluyeron 17 pacientes (34 amígdalas), mayores de 5 años sin antecedentes de riesgo, con indicación de amigdalectomía. A cada paciente, previo consentimiento informado y randomización, se le extrajo una amígdala con técnica fría y la contralateral con técnica electrobisturí monopolar. La percepción del dolor se midió con escala visual análoga (EVA) durante los primeros siete días posquirúrgicos. Paciente y familiares fueron enmascarados respecto a la técnica usada a cada lado.

Resultados: Se encontraron diferencias en la percepción del dolor entre los días 3 a $7(p<0,05)$ y al evaluarse la evolución del dolor en función del tiempo, ésta fue mayor con la técnica de electrobisturí (sin diferencias significativas en los 2 primeros días).

Conclusión: En los casos evaluados en este estudio, la amigdalectomía con técnica fría se asocia a menor dolor a contar del tercer día posquirúrgico.

Palabras clave: Amigdalectomia, dolor posoperatorio.

Médico. Departamento Otorrinolaringología, Pontificia Universidad Católica de Chile

2 Médico Epidemiología. Hospital Naval 


\section{ABSTRACT}

Introduction: There are different techniques for carrying out tonsillectomy. Among the most common ones are the "cold technique" (scalpel, scissors and handle), and the monopolar electrocautery technique. No consensus is available to define which technique is less painful.

Aim: To assess the effect of the surgical technique ("cold" vs monopolar electrocautery) on post-operative pain perception.

Material and method: Prospective blind randomized clinical trial. 17 patients with tonsillectomy indication (34 tonsils) over 5 years old, without major surgical risk, and after randomization and obtaining an informed consent, received a tonsillectomy using cold technique and the contra lateral side was removed using monopolar electrocautery technique. Pain perception was measured with visual analogue scale (VAS) during the first seven days after surgery. Patients and relatives were unaware of the technique used on each side.

Results: There were differences in pain perception between day 3 and $7(p<0.05)$. Assessing evolution across time, pain was higher with the electrocautery technique (no significant difference was found in the first 2 days).

Conclusion: Based on our results tonsillectomy using cold technique appears to be associated with less post-operative pain, starting on the third post-surgery day.

Key words: Tonsillectomy, Post-operative Pain.

\section{INTRODUCCIÓN}

La primera descripción de una técnica quirúrgica para realizar una amigdalectomía fue realizada por Aulus Cornelius Celsus, médico romano contemporáneo a Cristo el cual realizaba la extracción usando principalmente los dedos ${ }^{1}$. Pero no fue hasta fines del siglo XIX en que este procedimiento se hizo común, siendo hoy en día una de las cirugías más frecuente en la mayoría de los servicios de otorrinolaringología ${ }^{2}$. Existen muchas técnicas quirúrgicas descritas y un gran número de instrumentos diseñados con este fin, sin embargo no hay consenso que alguno de ellos sea claramente mejor ${ }^{1,3-6}$.

La frecuencia de complicaciones es baja, siendo su principal problema el dolor los días posteriores al procedimiento. El dolor posoperatorio es la causa más frecuente de retardo en la alimentación, prolongación de hospitalizaciones y motivo de consultas en servicios de urgencia².

Dos técnicas utilizadas habitualmente en el Servicio de Otorrinolaringología del Hospital Clínico de la Pontificia Universidad Católica son la técnica fría y la técnica mediante electrobisturí monopolar. En la técnica fría la disección es a través de bisturí frío, tijera y/o asa. En la segunda la disección es íntegramente por electrobisturí monopolar.

No existe consenso en este Centro o en la literatura, sobre cuál de las dos técnicas es mejor en términos de dolor posoperatorio.

El objetivo principal de este estudio es comparar la percepción de dolor mediante Escala Visual Análoga (EVA) entre estas dos técnicas al efectuarlas en un mismo paciente.

\section{MATERIAL Y MÉTODO}

Se realizó un ensayo clínico controlado, randomizado y ciego.

El universo consideró pacientes con indicación de amigdalectomía ya fuese por amigdalitis recurrente 0 por hiperplasia amigdalina obstructiva, mayores de 5 años de edad, que al momento de la entrevista clínica prequirúrgica pareciesen capaces de distinguir la localización de dolor en el cuello (qué lado les dolía) y que no tuviesen otros antecedentes mórbidos o factores de riesgos (categoría de riesgo prequirúrgico anestésico ASA I). Se consideraron como criterios de exclusión la exis- 
tencia de patología de base, nivel cognitivo-intelectual alterado, rechazo consentimiento informado y asimetría amigdalina.

Se invitó a participar a pacientes con las características descritas que consultaron en el Departamento de Otorrinolaringología de la Pontificia Universidad Católica de Chile entre enero y septiembre de 2009. Se obtuvo consentimiento informado (aprobado previamente por el Comité de Ética Científico de la Facultad de Medicina de la Pontificia Universidad Católica de Chile) de todos los pacientes y sus familias incluidos en el estudio durante la entrevista prequirúrgica

En cada paciente se procedió a realizar amigdalectomía con una de las técnicas (fría o con electrobisturí) en una amígdala, y con la técnica restante en la amígdala contralateral. Previo a la cirugía se realizó randomización simple para programar qué amígdala derecha o izquierda, sería extraída con cada técnica. Paciente y sus familiares fueron enmascarados, sin saber a qué lado se realizó cada técnica.

La técnica con electrocauterio comienza traccionando la amígdala con pinza Allis. Luego usando el electrobisturí con punta tipo cuchillo (paleta) con una potencia de 15W en coagulación, se diseca la amígdala por el plano capsular hasta su extracción completa.

En la técnica fría el polo superior de la amígdala es traccionado con pinza Allis. Con bisturí frío hoja N15 se incide la mucosa del polo superior hasta el plano capsular, luego con tijera Metzembaun se diseca respetando el plano. La extracción se completa usando asa para el polo inferior.

En ambos casos para la hemostasia se usó compresión con gasa con subgalato y de ser necesario electrocauterio dirigido específicamente a los puntos sangrantes.

La recolección de datos se inició el día siguiente de la cirugía, al momento del alta. Mediante Escala Visual Análoga (EVA) pediátrica (Figura 1, en menores de 15 años) 0 de adulto (Figura 2, en mayores de 16 años) según cada caso, se consultó la intensidad de dolor a cada lado del cuello. Cada paciente se llevó estas escalas de dolor a su domicilio, con el objetivo de repetir el registro de dolor diariamente durante los primeros siete días posoperatorios. Los resultados se entregaron en el primer control posoperatorio luego de siete días.

Para el análisis de resultados se consideró como variable dependiente, el dolor medido a través de EVA. La variable independiente fue la técnica quirúrgica (fría vs monopolar). También fueron mediadas la variables edad, indicación quirúrgica y presencia de sangrado severo perioperatorio.

En el análisis estadístico descriptivo se utilizaron promedios, desviación estándar (DE), medianas y porcentajes según cada variable. En el análisis analítico se usó test de Wilcoxon MannWhitney para muestras pareadas y ecuaciones de estimación generalizadas (GEE) para evaluar el dolor en función del tiempo. Se utilizó programa estadístico SPSS 16.0 y STATA 9.0.

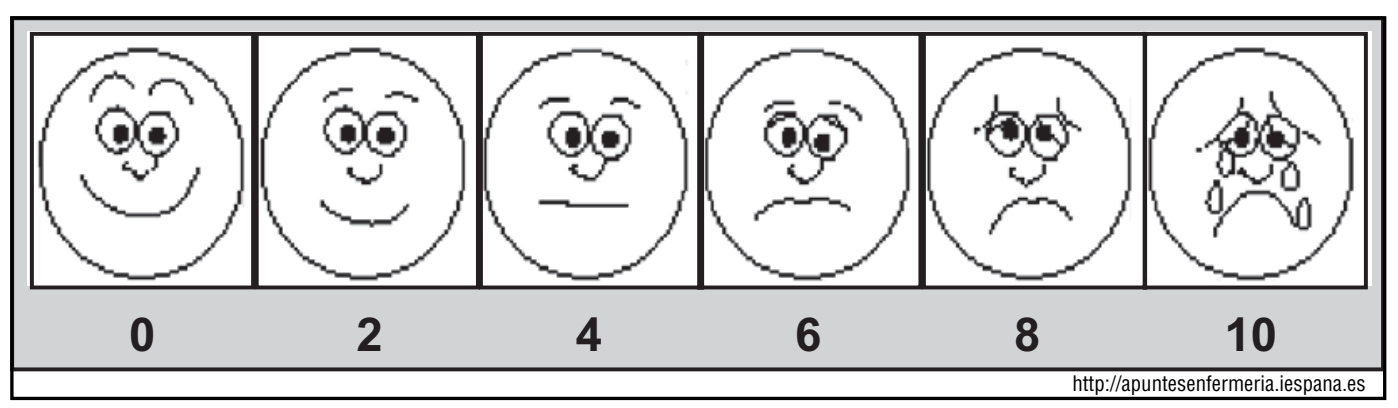

Figura 1. Escala Visual Análoga Pediátrica. Siguiendo la pauta del esquema, se pidió a los pacientes que registraran día a día, los primeros 7 días posoperatorios la intensidad del dolor que experimentaban en cada lado derecho e izquierdo. (La técnica utilizada en cada lado era desconocida para el paciente y su familia). Se utilizó esta escala en los menores de 15 años. 


\section{Escala analógica visual del dolor}

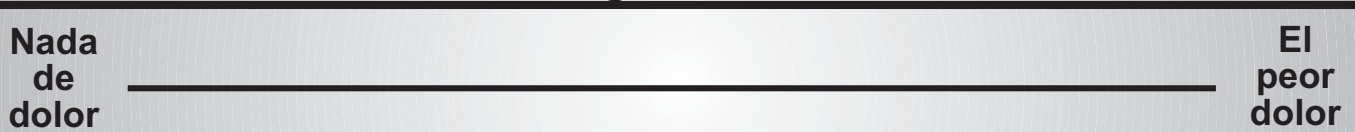

Instrucciones: Pida al paciente que indique en la línea en dónde está el dolor en relación con los dos extremos. Esta calificación es sólo una aproximación, por ejemplo, una marca en el medio indicaría que el dolor es aproximadamente la mitad del peor dolor posible.

http://apuntesenfermeria.iespana.es

Figura 2. Escala Visual Análoga Adulto. Siguiendo la pauta del esquema, se pidió a los pacientes que registraran día a día, los primeros 7 días posoperatorios la intensidad del dolor que experimentaban en cada lado derecho e izquierdo. (La técnica utilizada en cada lado era desconocida para el paciente y su familia). Se utilizó esta escala en los mayores de 16 años.

\section{RESULTADOS}

La muestra consideró 17 pacientes (34 amígdalas), entre 5 y 25 años de edad. La edad promedio fue de 13 años (DE 7,32) y la mediana de 11 años. $47 \%$ (8/ 17) correspondieron a género femenino. En $71 \%$ de los pacientes la indicación quirúrgica fue hiperplasia amigdalina, mientras que el $29 \%$ restante tuvo por diagnóstico amigdalitis recurrente. No hubo ningún caso de sangrado importante en esta serie.

En la Figura 3 podemos apreciar la evolución del dolor para cada técnica a lo largo de los 7 días (se muestran promedios de la escala EVA para cada día). Como detallamos en la Tabla 1, a partir del día 3 en adelante, la técnica fría muestra un promedio de dolor significativamente menor que la técnica con electrobisturí ( $p<0,05$ en test de Wilcoxon Mann-Whitney) al comparar ambos promedios dentro del mismo día.

Luego, al evaluar el comportamiento del dolor según escala EVA en el tiempo para cada técnica utilizando análisis de curvas GEE, se confirma una diferencia estadísticamente significativa a favor de la técnica fría $(p=0,0394)$.

\section{DISCUSIÓN}

Los datos en nuestra serie parecen demostrar que a partir del tercer día posoperatorio el uso de la técnica fría con bisturí, tijera y asa, se relacionaría con una menor intensidad de dolor posoperatorio.

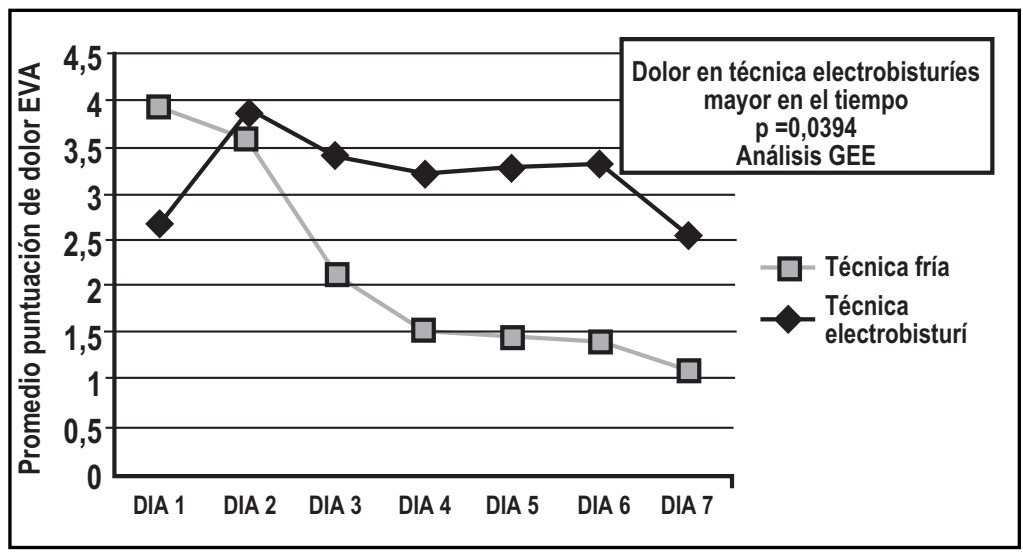

Figura 3. Promedio de dolor según técnica quirúrgica primeros 7 días posoperatorio. Día a día se muestra (para cada tipo de técnica quirúrgica implementado) el promedio de dolor según escala EVA. Se puede apreciar que a partir del tercer día el dolor manifestado en la amígdala operada con técnica fría es menor que con técnica electrobisturí (las diferencias durante los dos primeros días no son estadísticamente significativas, como se puede apreciar en la Tabla 1). En análisis GEE para evolución de promedios en el tiempo, confirma que la técnica fría presenta menor dolor en los primeros 7 días posoperatorio $(p=0,039)$. 
Tabla 1. Detalle de evaluación de dolor EVA según día y técnica quirúrgica

\begin{tabular}{|c|c|c|c|c|c|c|c|}
\hline & Técnica & Media & $\mathrm{DE}$ & Min & Max & Mediana & $P^{*}$ \\
\hline \multirow[t]{2}{*}{ Día 1} & Fría & 3,94 & 2,73 & 0 & 8 & 4 & 0,172 \\
\hline & electrobisturí & 2,71 & 2,71 & 0 & 8 & 2 & \\
\hline \multirow{2}{*}{ Día 2} & Fría & 3,59 & 2,55 & 0 & 8 & 4 & 0,885 \\
\hline & electrobisturí & 3,88 & 2,18 & 2 & 8 & 4 & \\
\hline \multirow{2}{*}{ Día 3} & Fría & 2,12 & 1,9 & 0 & 6 & 2 & ${ }^{*} 0,0396$ \\
\hline & electrobisturí & 3,41 & 1,87 & 1 & 8 & 3 & \\
\hline \multirow[t]{2}{*}{ Día 4} & Fría & 1,53 & 1,37 & 0 & 4 & 2 & ${ }^{\star} 0,0012$ \\
\hline & electrobisturí & 3,24 & 1,95 & 0 & 8 & 2 & \\
\hline \multirow[t]{2}{*}{ Día 5} & Fría & 1,47 & 1,28 & 0 & 4 & 2 & ${ }^{*} 0,0012$ \\
\hline & electrobisturí & 3,29 & 1,76 & 2 & 8 & 2 & \\
\hline \multirow[t]{2}{*}{ Día 6} & Fría & 1,41 & 1,97 & 0 & 7 & 1 & ${ }^{\star} 0,0036$ \\
\hline & electrobisturí & 3,35 & 2,4 & 0 & 9 & 2 & \\
\hline \multirow[t]{2}{*}{ Día 7} & Fría & 1,12 & 1,69 & 0 & 5 & 0 & ${ }^{*} 0,0086$ \\
\hline & electrobisturí & 2,59 & 2,6 & 0 & 9 & 2 & \\
\hline
\end{tabular}

*Diferencia estadísticamente significativa en test Wilcoxon Mann-Whitney.

Podemos apreciar una importante dispersión en las puntuaciones de dolor los primeros dos días posoperatorios. Luego del tercer día, la diferencia alcanza valores estadísticamente significativos (considerando un error alpha de 0,05) a favor de la técnica fría.

Creemos que nuestra metodología permite limpiar las variables de confusión habituales, al ser siempre el mismo paciente en el que se evalúan y contrastan ambas técnicas quirúrgicas. Cada grupo de 17 amígdalas en cada técnica quirúrgica comparte las propiedades de los mismos 17 pacientes, por lo que se eliminan variables de confusión como género, tolerancia al dolor de cada paciente, factores constitucionales, dificultad quirúrgica dada por la anatomía del paciente, entrenamiento del cirujano, dosis y tipo de analgésicos, uso de antibióticos, medicamentos durante la anestesia, etc. Así ambos grupos (amígdalas operadas con electrobisturí vs amígdalas operadas con técnica fría) son perfectamente comparables.

Sin embargo reconocemos como un posible sesgo del estudio la preferencia habitual y experiencia de cada cirujano con cada técnica en particular. Sin embargo varios cirujanos participaron indistintamente en el estudio, incluyendo residentes, aprendiendo en igual medida ambas técnicas.

Dentro de nuestros datos, es llamativa la gran dispersión en la calificación de la intensidad del dolor en el grupo de pacientes, probablemente las variables individuales como umbral de dolor y manejo anestésico perioperatorio son mucho más influyentes en el dolor durante las primeras 48 horas que la técnica quirúrgica utilizada.

Llama la atención el menor dolor mostrado por la técnica con electrobisturí el primer día posoperatorio (aunque dicha diferencia no fue estadísticamente significativa). Este fenómeno pudiese ser explicado por la destrucción por calor de terminales nerviosos libres en la zona, que al ser regenerados en los días siguientes, comienzan a aumentar la percepción del dolor, al igual como ocurre en cierto tipo de quemaduras.

Existen muchas técnicas quirúrgicas avaladas y utilizadas internacionalmente. La elección de cuál usar pasa en la actualidad por un sinfín de factores asociados, desde preferencias personales hasta facilidades para su implementación en los distintos centros quirúrgicos. Creemos que este trabajo sirve como un antecedente más al momento de elegir una técnica.

Este trabajo no cuenta con fuentes de financiamiento externa. Estudio realizado en el Hospital Clínico de la Pontificia Universidad Católica. Este trabajo cuenta con la aprobación del Comité de Ética de la Pontificia Universidad Católica de Chile (Resolución C.E. \# 66/09 fecha 19-3-2009). 


\section{BIBLIOGRAFÍA}

1. CURTIN JM. The history of tonsil and adenoid surgery. Otol Clin North Am 1987; 20: 415-21.

2. Núnez DA, Provan J, Crawford M. Postoperative tonsillectomy pain in Pediatric Patient. Arch Otolaryngol Head Neck Surg 2000; 126: 83741.

3. LeINBACH R. Hot versus cold tonsillectomy: A systematic review of the literature. Otolaryngol Head Neck Surg 2003; 129: 360-4.

4. Mann DG, St George C, Scheiner E, Granoff D, IMBER P. Tonsillectomy some like it hot. Laryngoscope 1984; 94: 677-9.

5. Koempel JA. On the origin of tonsillectomy and the dissection method. Laryngoscope 2002; 112: 1583-6.
6. Esteban F, Soldado M. Tonsillectomy by electrical dissection versus blunt dissection: a study of 838 cases (randomized trial of 207 cases and non-randomized prospective study of 631 cases). Acta Otorrinolaringol Esp 1998; 49(7): 541-7.

7. WeXLER DB. Recovery after tonsillectomy: electrodissection versus sharp dissection techniques. Otolaryngol Head Neck Surg 1996; 114: 576-81.

8. TAY H. Post-operative morbidity in electrodissection tonsillectomy. J Laringol Otol 1995; 109: 209-11.

9. Linden Be, Gross CW, Long te, Lazar RH. Morbidity in pediatric tonsillectomy. Laryngoscope 1990; 100(2 Pt 1): 120-4. 